\title{
Dislocations, Phason Defects, and Domain Walls in a One-Dimensional Quasiperiodic Superstructure of a Metallic Thin Film
}

\author{
Ph. Ebert, ${ }^{1,2}$ K.-J. Chao, ${ }^{1}$ Q. Niu, ${ }^{1}$ and C. K. Shih ${ }^{1}$ \\ ${ }^{1}$ Department of Physics, University of Texas at Austin, Austin, Texas 78712 \\ ${ }^{2}$ Institut für Festkörperforschung, Forschungszentrum Jülich GmbH, 52425 Jülich, Germany
}

(Received 16 April 1999)

\begin{abstract}
We investigated disorder and structural defects in a one-dimensional quasiperiodic superstructure of a thin Ag film on GaAs(110) surfaces by scanning tunneling microscopy. The superstructure forms sequences with long and short separations exhibiting a self-similarity. We demonstrate that the modulation can be described best with a Fibonacci sequence and deviations are due to structural defects. We identify dislocations, phason defects, and domain walls. The static stress field of dislocations is found to be a source of phason defects.
\end{abstract}

PACS numbers: 61.44.Br, 61.16.Ch, 68.35.Dv

Quasicrystals possess a well-ordered nonperiodic arrangement of atoms with long range rotational symmetries. This unique structure has attracted considerable attention and its detailed determination has been the issue of numerous studies. Deviation from the ideal quasicrystal structure, i.e., structural defects and disorder in quasicrystals, affect, however, to a large degree their properties, such as heat conduction, diffusion constants, phase transitions, and electron transport properties [1-8]. Therefore knowledge about defects and their relation to the quasiperiodic structure is a key to the understanding of quasicrystals and possibly even of their formation. To date defects have been investigated experimentally nearly exclusively in three- and two-dimensional quasicrystals $[2,9,10]$ by transmission electron microscopy. To our knowledge disorder and defects in one-dimensional quasicrystals have been addressed only theoretically, likely due to the lack of suitable model systems for experimental real space investigations.

In this Letter we investigate structural defects and disorder on a new type of one-dimensional quasicrystal: a modulated thin $\mathrm{Ag}$ film on $\mathrm{GaAs}(110)$ surfaces. We demonstrate that the film exhibits a quasiperiodic superstructure with a self-similarity and analyze the defects present on the film in real space. We identify dislocations, phason defects, as well as domain walls, and we show that the stress field of dislocations give rise to phason defects.

The one-dimensional quasiperiodic system investigated here is a novel system distinctively different from the one-dimensional quasicrystals known so far [11]. The one-dimensional quasiperiodic structure was obtained by depositing 1.5 to $1.7 \mathrm{~nm}$ thick $\mathrm{Ag}$ layers at $135 \mathrm{~K}$ on freshly cleaved GaAs(110) surfaces in ultrahigh vacuum. Following the low temperature deposition, the films were annealed at room temperature and imaged by scanning tunneling microscopy in the constant-current mode. Using this procedure we always obtained flat twodimensional Ag films [12]. The Ag films all exhibited a one-dimensional height modulation, which lead to stripes with a corrugation of $0.05 \mathrm{~nm}$ in scanning tunneling microscopy images (Fig. 1) [13]. Figure 1 shows that the stripes are obviously not having all the same separation and do not form an obvious periodic superstructure. We rather observed two different basic separations of the stripes of about $1.2 \pm 0.2$ and $1.7 \pm 0.2 \mathrm{~nm}$ [visible in Fig. 1(b)]. In the following we call these short $(S)$ and long $(L)$ segments, respectively. Figure 1(a) shows that at lower magnifications the STM images exhibit again stripes similar to those in Fig. 1(b). However, the stripes in Fig. 1(a) have significantly larger separations than the two basic stripes. The inflated stripes yield again a pattern similar to that of the basic stripes, only more complex. The large scale stripes appear due to an additional height modulation overlapping with the basic height modulation leading to the $L$ and $S$ segments. Figure 1(c) shows the Fourier transform of the stripes. The peaks in the Fourier transform can be well described and indexed [14] on the basis of two basic units, whose length scale by the golden mean in the reciprocal space. The peaks above $1 / S$ are connected with the substructure of the stripes, and they again exhibit features scaled by the golden mean (e.g., see peak at 1.6/S). All peaks exhibit approximately the same line width in all directions of the Fourier space. From the Fourier transform as well as from the real space images we found that the ratio of the basic long and short separations in STM images is $1.55 \pm 0.15$. This ratio agrees within the error margin with that expected for the two basic units of the Fibonacci sequence $\left[\tau=\frac{1}{2}(\sqrt{5}+1)=1.618 \ldots\right]$. Furthermore, the self-similarity and the Fourier transform are also consistent with a quasiperiodic type of order.

The stripes form sequences of long and short segments. One sequence is indicated below Fig. 1(b). We analyzed a large number of such sequences (with lengths of up to 135 segments and a total number of segments of over 1500 taken from different Ag films) statistically to determine which structural model agrees best with our observation. This is a necessary basis for the analysis of the defects 

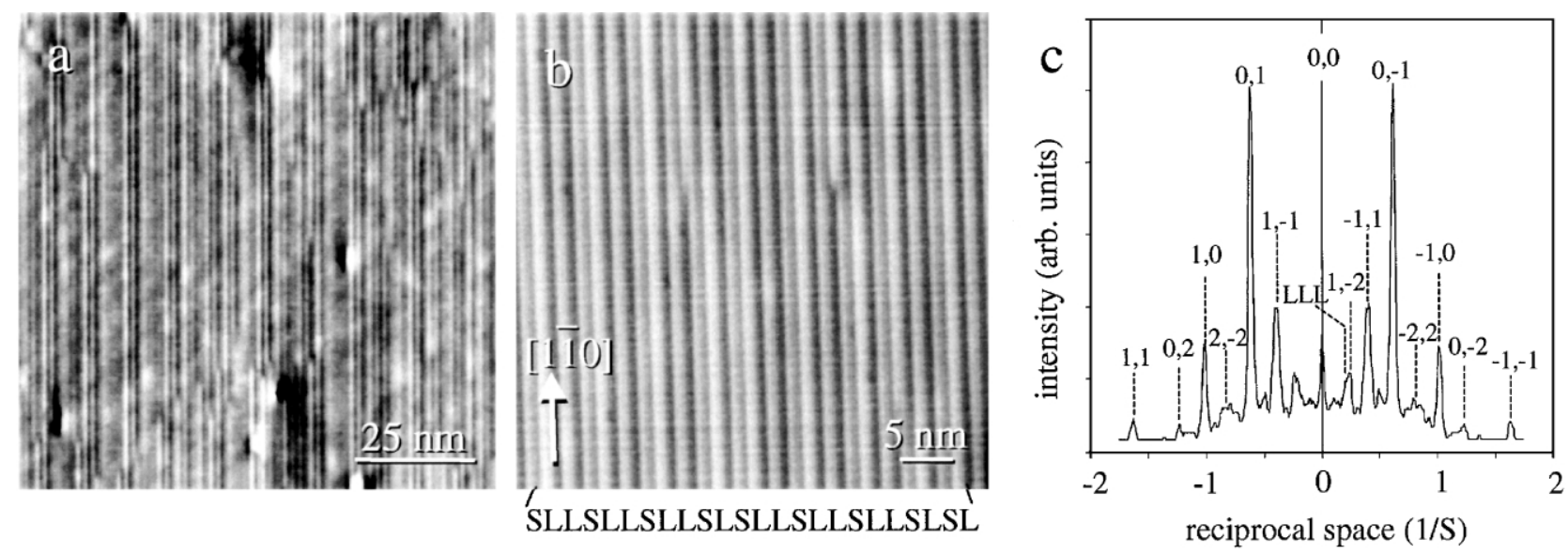

FIG. 1. Scanning tunneling microscopy constant-current images of the superstructure of a $1.5 \mathrm{~nm}$ thick Ag film grown at low temperature on GaAs(110) surfaces and annealed at room temperature. Shown are two magnifications. At higher magnification in (b) stripes with the basic long $(L)$ and short $(S)$ separations are observed, whereas at lower magnification (a) stripes with inflated long and short separations are found. Frame (c) shows the Fourier transform indexed according to Ref. [14].

and the disorder. We compared all sequences of a certain length $l$ with all possible sequences of the same length cut from calculated periodic and quasiperiodic structures. Figure 2 shows as a function of the length of the sequence $l$ the fraction of sequences not matching any part of a theoretical sequence. The comparison with a Fibonacci sequence as well as with LLS and LLSLS periodic sequences (first two approximants to the Fibonacci sequence) shows that the experimentally observed data agree best with the golden-mean Fibonacci sequence, because it has the lowest number of nonmatching sequences. No good agreement is found with the silver-mean quasiperiodic sequence suggested earlier [15]. If we define that the coherence length of the surface order is given by the length of the sequence with $50 \%$ nonmatching sequences, we obtain that

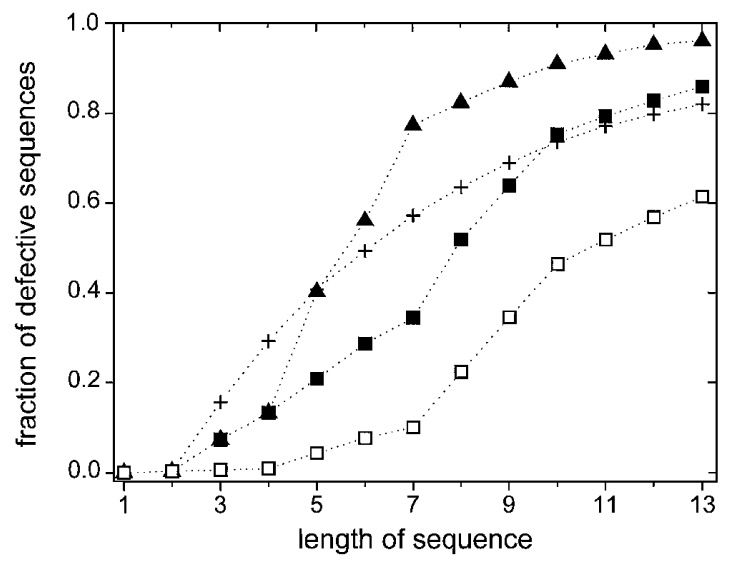

FIG. 2. Fraction of the observed sequences of length $l$ not matching with any sequence of the same length taken from the Fibonacci sequence (filled squares) as well as from the LLS (crosses) and LLSLS (filled triangles) approximants to the Fibonacci sequence. The open squares show the fraction of sequences of the measured data corrected for the missing short segment defect (see text) not matching with any sequence from the Fibonacci sequence. the longest coherence length is given for the Fibonacci sequence with about 8 segments, whereas the periodic approximant structures yield only a coherence length of 5.5 to 6 segments. Thus, taking this and the above shown self-similarity as well as ratio of long and short segments into account, the experimentally observed sequences of long and short separations are described best by a goldenmean quasiperiodic order with a coherence length of about $12.5 \mathrm{~nm}$. This conclusion agrees also with the low energy electron diffraction pattern, which can be explained well by a golden-mean quasiperiodic ordering. We note that a distinction between random tiling and a perfect quasiperiodic order is difficult in the present case, because both structures should yield sharp diffraction peaks. Similarly, we cannot rule out that the structure could be explained by an approximant sequence of high order. However, high order approximant structures and quasiperiodic structures differ only marginally, and a discrimination is beyond our statistical accuracy based on more than 1500 sequences for all length shown. Furthermore, as we discuss below, most of the limited coherence length is due to structural defects.

The limited coherence length indicates that there must be a number of defects present in the sequences. We found that the major defect is a missing short segment leading to three or sometimes more consecutive long segments. The importance of this type of defect is corroborated by the observation of dislocations correcting these triple long sequences into LLSL sequences as shown below. If we correct the sequences for this defect, we obtain a much better agreement with the Fibonacci sequence as shown by the open square data points in Fig. 2. Correcting only for this defect increases the coherence length to about $11 \mathrm{seg}-$ ments or $17 \mathrm{~nm}$. We note that the fraction of $L$ segments decreases from $66 \%$ to $62 \%$ by the correction yielding a very good agreement with that of the ideal Fibonacci sequence $(\approx 61.8 \%)$. This demonstrates that the missing short sequences are the major defects present. This result 


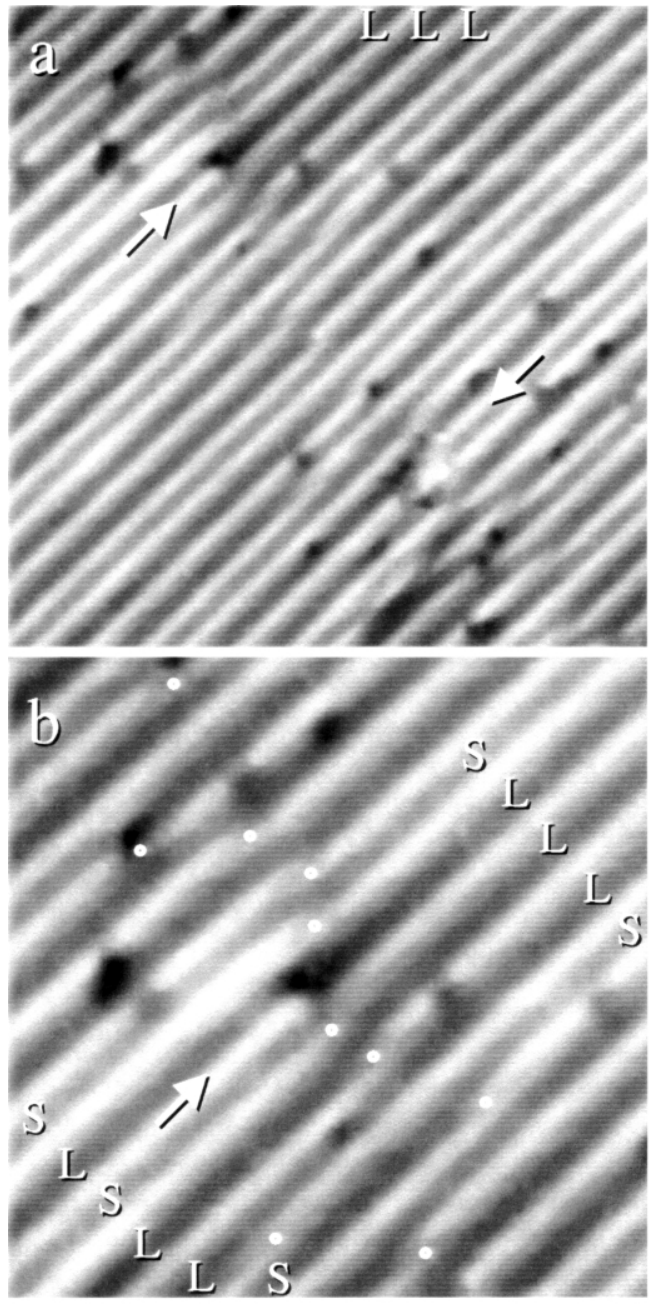

FIG. 3. (a) Constant-current scanning tunneling microscopy image showing a pair of dislocations with opposite Burgers vectors in the one-dimensional quasiperiodic superstructure. The arrows indicate the inserted rows. Frame (b) is a magnified image of the upper dislocation in frame (a). The dislocation inserts a short segment into three consecutive long segments, removing thus a domain wall present in the upper left part of the image. Close to the dislocation several phason defects are marked by points. Both images show the empty states.

is further corroborated by the fact that the LLL sequences appear also as shoulder in the Fourier transform in Fig. 1(c). We note that the corrected values do not agree well with the periodic approximant sequences. Figure 3(a) shows a STM image of such a LLL defect, which we can call either domain wall or grain boundary, in the upper right corner. The three consecutive long separations between the segments are marked LLL. At the position of the arrow one additional stripe is inserted, which exhibits a short separation to the neighboring stripe ( $S$ segment). We observed this feature with concentrations of approximately $5 \times 10^{10} \mathrm{~cm}^{-2}$. Each of these dislocations inserts a short segment into consecutive long segments. This supports our identification of the main defect in the observed sequences being missing short segments. The defects in Fig. 3 are dislocations in a one-dimensional quasicrystal. The observed dislocations have all Burgers vectors oriented parallel to the quasiperiodic direction [ $\langle 001\rangle$ direction of the $\mathrm{GaAs}(100)$ substrate] with a length of a short separation. We frequently observed pairs of dislocations with opposite Burgers vectors as shown in Fig. 3(a), although isolated dislocations were found, too.

In addition to the dislocations there are still other defects present which are responsible for the limited coherence length. Figure 4 shows the most frequent defect, a phason defect. A large degree of the disorder is due to phason defects. We observed on the surfaces phason defects with concentrations of about $10^{12} \mathrm{~cm}^{-2}$. Phason defects are by definition a localized displacement from a quasiperiodic lattice position to another neighboring position not within the quasiperiodic lattice. In a one-dimensional quasiperiodic sequence a phason defect reverses the long and short segments. This is exactly what the defects in Fig. 4 do as indicated by the long and short sequences.

We found that a significant part of the phason defects were not homogeneously distributed on the surface. The small circles in Fig. 3(b) show that close to the dislocation core a high concentration of phason defects occurs. The STM image already indicates that the phason defects mainly extend along the $\langle 001\rangle$ direction from the dislocation core. A statistical analysis of the distribution of phason defects close to dislocation cores is presented in Fig. 5. The increased concentration as well as the spatial localization along the $\langle 001\rangle$ direction of phason defects close to the dislocation core is obvious. In addition, we found that all phason defects shift the stripes away from the dislocation core. Thus dislocations induce oriented phason defects. The presence of phason defects can be understood in terms of stress relief close to the dis-

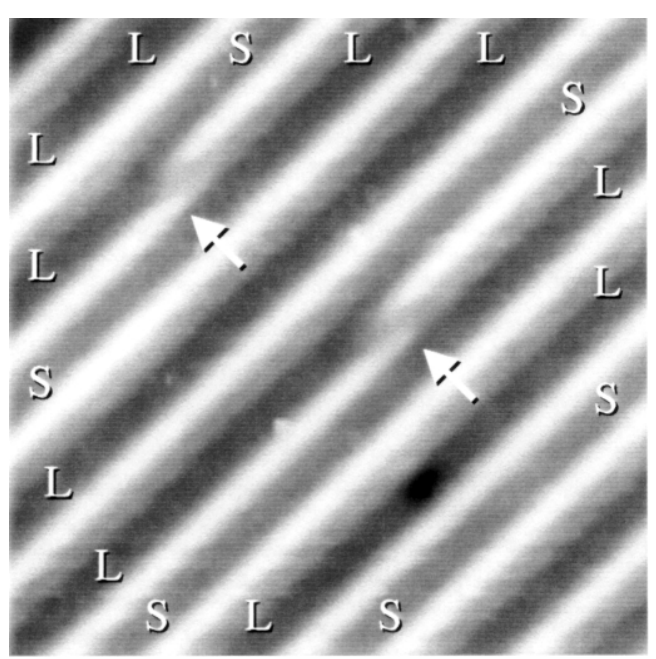

FIG. 4. Constant-current scanning tunneling microscopy image (empty states) of two phason defects (marked by the arrows) in the one-dimensional quasiperiodic superstructure. The phason defects reverse a long-short $(L S)$ to a short-long $(S L)$ combination. 
location core, because the phason defects lead to the increase in separation necessary for the insertion of the additional stripe by the dislocation. We note that in our case the static stress field around a dislocation is a source of phason defects, whereas earlier work in icosahedral quasicrystals reported that the motion of dislocations induces phasons and disorder [16,17].

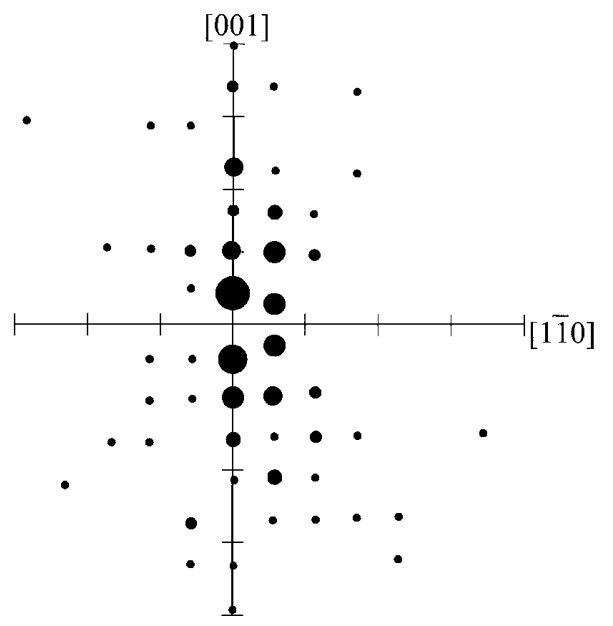

FIG. 5. Distribution of phason defects close to dislocation cores. The size of the points is proportional to the number of phason defects per surface area observed (ranging from 1 to 8 ) around 14 dislocations. The data show that the concentration of phason defects is considerably increased close to the dislocation core, and most phason defects are arranged along the $\langle 001\rangle$ direction, but none are found along the $\langle 110\rangle$ direction. The subdivisions along the axes indicate a distance of $3 \mathrm{~nm}$. The distances are measured from the core of the dislocation in the center of the graph. The inserted row of the dislocation is on the left side of the $x$ axis.

The STM images revealed even more types of defects. We found two consecutive short segments and long segments replacing short ones. These defects have, however, rather low concentrations, thus leading only to minor disorder.

Finally, we could obtain a quasiperiodic superstructure only for $\mathrm{Ag}$ on $\mathrm{GaAs}(110)$ substrates. Substrates with other lattice constants yield different superstructures. This suggests that the lattice parameters of $\mathrm{GaAs}(110)$ and $\operatorname{Ag}(111)$ scale such that it becomes energetically favorable to form a superstructure where defects adapt the superstructure order as closely as possible to the ideal quasiperiodic order. This would lead to the investigated "short" range quasiperiodic order interrupted by defects, which accommodate best the strain.

In conclusion, we investigated and identified for the first time in real-space by scanning tunneling microscopy the structural defects inducing the disorder in a onedimensional quasiperiodic superstructure of a thin Ag film on GaAs(110) surfaces. The superstructure exhibits a selfsimilarity with sequences consisting of a long and a short basic separation, whose length ratio is consistent with the golden mean. We demonstrated that the superstructure can be described best with a Fibonacci sequence modified by structural defects. The defects reducing the coherence length to about $12.5 \mathrm{~nm}$ are identified as dislocations, phason defects, and domain walls. The static stress field of dislocations is found to be a source of phason defects.

This work was supported by NSF Grant No. DMR9705406, NSF Science and Technology Center Grant No. CHE-8920120, Texas Advanced Research Program, and the Welch Foundation. P. E. thanks F. Kluge for helpful discussions and the Deutsche Forschungsgemeinschaft for financial support under Grant No. UR51/3-2.

[1] C. Janot, Quasicrystals: A Primer (Oxford University Press, Cambridge, 1994), 2nd ed.

[2] L. Beraha, M. Duneau, H. Klein, and M. Audier, Philos. Mag. A 78, 345 (1998).

[3] T. Klein, C. Berger, D. Mayou, and F. Cyrot-Lackmann, Phys. Rev. Lett. 66, 2907 (1991); D. Mayou, C. Berger, F. Cyrot-Lackmann, T. Klein, and P. Lanco, Phys. Rev. Lett. 70, 3915 (1993).

[4] T. Dotera and P. J. Steinhardt, Phys. Rev. Lett. 72, 1670 (1994).

[5] M. de Boissieu, M. Boudard, B. Hennion, R. Bellissent, S. Kycia, A. Goldman, C. Janot, and M. Audier, Phys. Rev. Lett. 75, 89 (1995).

[6] R. Blüher, P. Scharwaechter, W. Frank, and H. Kronmüller, Phys. Rev. Lett. 80, 1014 (1998).

[7] M. Krajčí, J. Hafner, and M. Mihalkovič, Phys. Rev. B 56, 3072 (1997).

[8] T. Fujiwara, S. Yamamoto, and G. Trambly de Laissardière, Phys. Rev. Lett. 71, 4166 (1993).

[9] M. Wollgarten, M. Beyss, K. Urban, H. Lieberts, and U. Köster, Phys. Rev. Lett. 71, 549 (1993).

[10] Y. Yan, S. J. Pennycook, and A. P. Tsai, Phys. Rev. Lett. 81, 5145 (1998).

[11] L. X. He, X.Z. Li, Z. Zhang, and K. H. Kuo, Phys. Rev. Lett. 61, 1116 (1988).

[12] A. R. Smith, K.-J. Chao, Q. Niu, and C.-K. Shih, Science 273, 226 (1996).

[13] The presently available data suggest that the modulations are variations in the film thickness, induced by different lattice parameters of $\mathrm{GaAs}(110)$ and $\mathrm{Ag}(111)$.

[14] P. Mikulík, in Beyond Quasicrystals, edited by F. Axel and D. Gratias (Les Edition de Physique, Les Ulis, 1995).

[15] Holes or pits in the Ag film modify and pin the superstructure, such that different length scales and sequences appear. Here we analyzed only complete Ag layers with no holes in contrast to Ref. [12].

[16] M. Feuerbacher, C. Metzmacher, M. Wollgarten, K. Urban, B. Baufeld, M. Bartsch, and U. Messerschmidt, Mater. Sci. Eng. A 226-228, 943 (1997); R. Wang, M. Feuerbacher, W. Yang, and K. Urban, Philos. Mag. A 78, 273 (1998).

[17] R. Mikulla, P. Gumbsch, and H.-R. Trebin, Philos. Mag. Lett. 78, 369 (1998); R. Mikulla, J. Stadler, F. Krul, H.-R. Trebin, and P. Gumbsch, Phys. Rev. Lett. 81, 3163 (1998). 